\title{
Zwischen Neopaternalismus und Repression
}

\begin{abstract}
Nicht einmal die Hälfte aller Beschäftigten in Deutschland ist durch einen Betriebsrat vertreten. Der Beitrag fragt nach den Gründen, warum der Betriebsrat in vielen Betrieben nicht als effektive Form der Interessenvertretung ins Spiel kommt. Welche Bedingungen und Konstellationen prägen diese Betriebe? Wie ist die Interaktion zwischen Unternehmensleitung und Beschäftigten beschaffen? Warum machen die Beschäftigten von ihrem Recht, einen Betriebsrat zu gründen, keinen Gebrauch?
\end{abstract}

\section{Einleitung}

Das System der industriellen Beziehungen in Deutschland ist geprägt durch die Arbeitsteilung von Betriebsräten und Gewerkschaften. In den letzten beiden Jahrzehnten hat sich dieses System jedoch als brüchig erwiesen. Einerseits nimmt die Zahl der Unternehmen, die an einen Flächentarifvertrag gebunden sind, kontinuierlich ab. Andererseits verfügt nur jeder zehnte Betrieb über einen Betriebsrat, nicht einmal die Hälfte aller Beschäftigten in der privaten Wirtschaft ist in Deutschland tatsächlich durch einen Betriebsrat vertreten; und es deutet sich inzwischen an, dass diese Anzahl weiter abnimmt (Ellguth/Kohaut 2007). Durch aktuelle Entwicklungen wird diese Bruchstelle immer stärker belastet. Einerseits gewinnt die betriebliche Ebene durch die Dezentralisierung der Tarifpolitik zunehmend an Bedeutung. Andererseits finden sich Betriebe ohne Betriebsrat gerade in solchen Bereichen, die durch die Entwicklung zu einer globalisierten Dienstleistungsgesellschaft an Bedeutung gewinnen: im Bereich der Wissensarbeit ebenso wie in der prekären Dienstleistungsarbeit.

\section{Das Münchener Forschungs- projekt zu Betrieben ohne Betriebsrat}

Der Lehrstuhl für Soziologie an der Technischen Universität München hat deshalb in einem dreijährigen Forschungsprojekt ${ }^{1}$ die Arbeitsbeziehungen in Betrieben ohne Betriebsrat anhand einzelner Fallstudien untersucht. Insgesamt wurden Fallstudien in 26 Unternehmen durchgeführt, die sich zu gleichen Teilen auf drei Sektoren verteilen: wissensbasierte Unternehmen der „New Economy" (insbesondere der Software-Industrie und der Systembetreuung), klassische Dienstleistungsunternehmen (vom Einzelhandel bis zur Alten- und Krankenpflege) sowie mittelständische Industrieunternehmen (vom Maschinenbau bis zum Möbelhersteller). Ursprünglich wollten wir uns auf größere Betriebe ab etwa $100 \mathrm{Be}$ schäftigten beschränken. Es hat sich aber bald herausgestellt, dass eine Konstellation für unsere Fragestellung besonders relevant ist und deshalb nicht vernachlässigt werden darf: kleine Filialbetriebe großer international aufgestellter Unternehmen wie Discounter und Fast-Food-Ketten.

In den meisten dieser Unternehmen haben wir uns auf Kurzfallstudien beschränkt. Fünf Unternehmen haben wir für Intensiv-Fallstudien ausgewählt, bei denen wir eine größere Anzahl an Interviews mit Beschäftigten verschiedener Hierarchieebenen und verschiedener Betriebe des gleichen Unternehmens geführt haben: ein Software-Unternehmen mit 900 Beschäftigten, ein Maschinenbau-Unternehmen mit 120 Beschäftigten, eine KFZ-Reparaturkette, die deutschlandweit ca. $11.000 \mathrm{Be}$ schäftigte hat, ein Paketdienst mit 14.000 Beschäftigten in Deutschland und eine Discounter-Kette mit geschätzten $80.000 \mathrm{Be}$ schäftigten. In den letzten drei Fällen gibt es im Unternehmen auch Betriebe mit Betriebsrat, sodass wir in diesen Fällen die Situation in Betrieben mit und ohne Betriebsrat vergleichen konnten.

Die Auswertung der Fallstudien erfolgte unter der Fragestellung: Welcher Logik folgt die Interaktion zwischen Unternehmensleitung und Beschäftigten? Unter welchen Bedingungen ergeben sich stabile soziale Beziehungen, die einen Betriebsrat (auch aus Sicht der Beschäftigten) als unnötig oder gar als Störfaktor erscheinen lassen? Wo gibt es Bruchstellen, die im Konfliktfall doch zur Gründung eines Be- triebsrats führen können? Aus den 26 Unternehmensfallstudien haben sich dabei vier Konstellation von Betriebsratslosigkeit herauskristallisiert, die durch typische Interaktionsmuster zwischen Beschäftigten und Unternehmensleitung geprägt sind.

(1) In wissensbasierten Unternehmen der New Economy beruht die Interaktion zwischen Beschäftigten und Unternehmensleitung auf dem Wechselspiel von Einbindung und Autonomie. Die Beschäftigten legen Wert auf ihre Autonomie, und die Personalführung zielt darauf ab, diese $\mathrm{Au}$ tonomie in einer für das Unternehmen produktiven Weise einzubinden. Die Arbeitsbeziehungen sind durch einen NeoPaternalismus geprägt, dessen postmoderne Patchwork-Familie erstaunliche Parallelen zum klassischen Familienunternehmen aufweist. Betriebsräte gelten hier oft als Relikte der industriellen Vergangenheit, die die Tendenz haben, die Autonomie der Beschäftigten (etwa im Hinblick auf die Arbeitszeit) einzuschränken.

(2) Einen zweiten Bereich bilden hochspezialisierte mittelständische Industrieunternehmen im ländlichen Raum. Hier sind die Arbeitsbeziehungen durch die Reziprozität gegenseitiger Anerkennung geprägt. In solchen Unternehmen setzt das Management bewusst auf informelle Formen der Inte-

\footnotetext{
1 Das Projekt wurde von der Deutschen For schungsgemeinschaft (DFG) gefördert.

Stefan Lücking, Dr., wissenschaftlicher Mitarbeiter, Lehrstuhl für Soziologie, TU München. Arbeitsschwerpunkte: Europäische Arbeitsbeziehungen, prekäre Interessenvertretung, Soziologie der Wissensarbeit.

e-mail: stefan.luecking@wi.tum.de
} 
ressenaushandlung, weil es befürchtet, dass die Existenz eines Betriebsrats seine Fähigkeit einschränken könnte, schnell und flexibel auf „den Markt" reagieren zu können. Die Beschäftigten lassen sich auf solche informellen Arrangements ein, solange sie den Eindruck haben, dass die Vorteile für sie überwiegen.

(3) Ein drittes Interaktionsmuster lässt sich keiner bestimmten Branche zuordnen, wohl aber einer bestimmten Eigentümerstruktur: es ist typisch für ehemalige Familienunternehmen, die in den Besitz anonymer Investoren übergegangen sind. Hier trifft ein patronaler Führungsstil auf eine pragmatische Haltung der Beschäftigten. Die Unternehmenskultur ist noch patriarchal geprägt, nur ist aufgrund des finanziellen Drucks der Investoren der paternalistische Aspekt der Fürsorge weitgehend abhanden gekommen. Die Beschäftigten richten sich pragmatisch in dieser Situation ein, weil ihnen diese Unternehmen einerseits Aufstiegsmöglichkeiten bieten, die sie mit ihrer Qualifikation anderswo nicht haben, und andererseits die Gründung eines Betriebsrats als Tabubruch gilt - mit allen Konsequenzen.

(4) Im Bereich prekärer Dienstleistungsarbeit verstärken sich Repression und Ohnmacht gegenseitig. Hier treffen Beschäftigtengruppen, die auf solche „Jobs“ angewiesen sind und sich entsprechend ohnmächtig fühlen, auf eine Führungskultur, die jede Form von Interessenartikulation unterdrückt und die Gründung von Betriebsräten mit allen Mitteln verhindert.

Bei diesen Konstellationen handelt es sich um Idealtypen, um Abstraktionen, die zwischen einzelnen Fallstudien charakteristische Gemeinsamkeiten herausarbeiten, die sie von anderen Fallstudien abgrenzen. Zwar kann es in Einzelfällen auch Überschneidungen geben. So gibt es z. B. drei Fälle, in denen ein autokratischer Führungsstil (der eher dem Muster „Patronage Pragmatismus" entspräche) auf hochqualifizierte und auf Autonomie bedachte Beschäftigte trifft (Böhm/Lücking 2006, S. 129-132). Aber jedes der Interaktionsmuster ist auf der Basis von vier bis fünf Fällen gebildet, sodass sich 18 der 26 Fallstudien eindeutig einem dieser Interaktionsmuster zuordnen lassen.

Gemeinsam ist diesen vier Konstellationen, dass es sich um jeweils typische Fa- cetten einer globalisierten Wirtschaft handelt. Wissensarbeit und prekäre Dienstleistungsarbeit stellen die beiden Extreme der aktuellen Entwicklung dar, die in der Literatur am häufigsten diskutiert werden. Die hochspezialisierten Industrieunternehmen haben sich gerade aufgrund ihrer geringen Größe und der damit verbundenen Flexibilität erfolgreich in globale Marktnischen eingenistet. An der dritten Konstellation, den ehemaligen Familienunternehmen, zeigt sich, was passiert, wenn eine traditionell patriarchale Unternehmenskultur auf die engen finanziellen Vorgaben von Private-Equity-Gesellschaften und anderen anonymen Investoren trifft.

\section{Einbindung und Autonomie in wissensbasierten Unternehmen}

Der Interaktionsmodus, den wir in wissensbasierten Unternehmen vorgefunden haben, lässt sich als Wechselspiel von Einbindung und Autonomie beschreiben. In diesen Unternehmen treffen hochqualifizierte Beschäftigte mit einem hohen intrinsischen Interesse an ihrer Arbeit auf ein dezidiertes und reflektiertes Human Resource Management. Autonomie ist ein hoher Wert, und die Personalführung ist darauf ausgerichtet, den Beschäftigten einerseits diese Autonomie-Spielräume zu ermöglichen und die Autonomie andererseits so einzugrenzen, dass sie für den wirtschaftlichen Erfolg des Unternehmens funktional wird.

Bei den Fallstudien dieses Typus handelt es sich größtenteils um Unternehmen der Softwareindustrie und angrenzender Dienstleistungen. Auf der Basis der Typologie von Boes und Baukrowitz lassen sie sich als „Lack-Turnschuh-Unternehmen“ (Boes/Baukrowitz 2002, S. 84-91) einordnen: Es handelt sich um Unternehmen, die im Rahmen der IT-Revolution neu gegründet wurden, aber aufgrund ihrer Größe und der formalen Leitungsstruktur den Status von Start-ups längst überwunden haben. Wie für den Bereich der Wissensarbeit üblich, handelt es sich um Unternehmen ,jenseits des Tarifsystems“ (Artus 2005, S. 395f.), für die „der Tarif“ nicht einmal als entfernter Bezugspunkt eine Rolle spielt, sondern vollständig durch „den Markt" ersetzt ist. Entgelte werden in der Regel individuell ausgehandelt, wobei es bei den Einstiegsgehältern je nach Marktlage zu erheblichen Unterschieden kommen kann. Arbeitszeiten sind völlig unreguliert. Phasen mit exzessiven Arbeitszeiten, etwa vor dem Abschluss von Projekten, wechseln mit ruhigeren Phasen. Dieser deregulierte Zustand ist Teil der „Autonomie" der Beschäftigten und wird von diesen zum Teil so gewünscht.

Aus Sicht des Managements gehören nicht nur Tarifverträge, sondern auch Betriebsräte einer anderen Welt bzw. einer anderen Zeit an. Die Führungskräfte sehen Betriebsräte als Relikt der Industrialisierung, die nur noch für solche Branchen Sinn machen, in denen die Beschäftigten leicht ersetzbar und deshalb auf eine kollektive Interessenvertretung durch Gewerkschaften und Betriebsräte angewiesen sind. Die hochqualifizierten Beschäftigten im Bereich der Wissensarbeit dagegen könnten ihre Interessen viel besser individuell durchsetzen und seien deshalb nicht auf kollektive Formen der Interessenvertretung angewiesen. Überhaupt spiele der duale Interessenkonflikt zwischen Arbeitnehmern und Arbeitgebern im Bereich der Wissensarbeit keine Rolle mehr. Stattdessen existiere ein wirres Gemenge individueller Interessenkonflikte. Aufgabe der Personalführung sei es, eine Balance zwischen diesen individuellen Interessen herzustellen.

Besonders ausgeprägt ist die Ablehnung eines Betriebsrats bei den Teilen des Managements, die wir dem „neopaternalistischen“ Orientierungsmuster zuordnen (vgl. Böhm/Lücking 2006, S. 114-118). Diese Personen verstehen Human Resource Management als persönliches Bemühen um das Wohl der Beschäftigten. Der Grundgedanke ist der, dass Wissensarbeiter und -arbeiterinnen ein intrinsisches Interesse an ihrer Arbeit haben und deshalb keine zusätzliche Motivation benötigen, sondern nur die richtigen Rahmenbedingungen, um erfolgreich und auch im Sinne des Unternehmens effektiv arbeiten zu können. Diese Strategie der Sorge um das Wohl der Beschäftigten impliziert, dass die Personalführung letztendlich am besten weiß, was im Interesse „ihrer Mitarbeiter“ ist. Aus dieser Sicht sind Betriebsräte nicht nur dysfunktional für eine individualisierte und auf Autonomie bedachte Belegschaft, sondern auch ein Affront gegenüber der Kompetenz der Personalleitung als Instanz des Interessenausgleichs. 
$\mathrm{Zu}$ den vom Management initiierten Formen der Konfliktlösung gehören auch alternative Gremien, die allerdings in der Regel keinen kontinuierlichen Charakter haben, sondern den von Arbeitsgruppen oder Task Forces, die ad hoc zur Lösung aktueller Probleme eingerichtet werden - in einem Fall etwa, als die Umstellung des Prämiensystems zu Unzufriedenheit unter den Beschäftigten führte. In den von uns geschilderten Fällen wurden die Mitglieder dieser Gremien von der Personalleitung benannt, die darauf achtet, dass Personen aus verschiedenen Abteilungen und von verschiedenen Hierarchieebenen beteiligt werden, die für die Lösung des Problems als relevant und kompetent erachtet werden und gewährleisten, dass das Ergebnis der Arbeitsgruppe von den übrigen Beschäftigten akzeptiert wird.

Das Fehlen eines Betriebsrates lässt sich in diesen Unternehmen allerdings weder durch die Ablehnung von Seiten des Managements noch allein durch die Existenz anderer Formen der Konfliktlösung erklären, wenngleich Letztere - wenn sie erfolgreich sind - dazu beitragen können, dass die Beschäftigten einen Betriebsrat als unnötig ansehen. Die Beschäftigten sind selbstbewusst genug, um im Falle einer Betriebsratsgründung einen Konflikt mit dem Management einzugehen. Und sie wissen genau, dass Konfliktlösungen im Rahmen der vom Management initiierten Ad-hocGruppen auf das Wohlwollen des Managements angewiesen sind und sich Konfliktlösungen gegen den Willen der Unternehmensleitung nur mit Hilfe eines Betriebsrates durchsetzen lassen.

Immerhin ist es in zwei der von uns untersuchten Unternehmen noch vor Abschluss des Projekts zu einer Betriebsratsgründung gekommen. In beiden Fällen waren weitreichende Restrukturierungen der Anlass, in dem einen Fall in Folge einer Fusion, in dem anderen Fall aufgrund der Reorganisation des europäischen Geschäftsbereichs. Beide Fälle zeigen, dass der Betriebsrat für die Beschäftigten stets eine Option bleibt, falls es zu Konflikten kommt, die sich nicht mehr auf freiwilliger Basis lösen lassen. Solange dieser Punkt nicht erreicht ist, haben auch die Beschäftigten kein Interesse an einem Betriebsrat.

Ein entscheidender Grund dafür liegt in der individuellen Berufsorientierung der Beschäftigten. Sie sind an ihrer Arbeit interessiert und wollen auf ihrem Tätigkeitsgebiet vorankommen. Ein kontinuierliches
Engagement in einem Gremium wie dem Betriebsrat stellt für diese Beschäftigten eine Belastung dar - verbunden mit der Befürchtung, auf dem Wissensgebiet, in dem sie sich spezialisiert haben, abgehängt zu werden. Die Schwelle, sich langfristig in einem Betriebsrat zu engagieren, ist dementsprechend hoch. Solange sie in der Vertretung ihrer individuellen Interessen erfolgreich sind und andere Formen der Konfliktlösung zu akzeptablen Ergebnissen führen, besteht für sie kein Anlass, sich für eine Betriebsratsgründung zu engagieren.

Ein weiterer Grund besteht darin, dass ein Teil der Beschäftigten der New Economy die Ansicht des Managements teilt, dass Betriebsräte genauso wie Gewerkschaften und Tarifverträge einer vergangenen Zeit angehören. Gewerkschaftsfeindliche Haltungen sind weit verbreitet und übertragen sich auch auf die Institution Betriebsrat. Unsere Interviews zeigen aber auch (ähnlich wie die Diskussionen im Internet um die Betriebsratsgründung bei SAP), dass ein anderer Teil der IT-Beschäftigten Gewerkschaften und Betriebsräten prinzipiell positiv gegenübersteht.

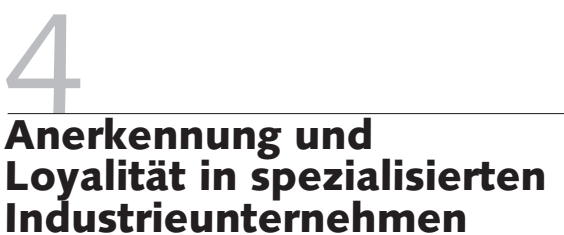

Prägend für den Interaktionsmodus Anerkennung/Loyalität ist die Reziprozität wechselseitiger Anerkennung im Rahmen einer Tauschbeziehung, wobei den betrieblichen Akteuren stets bewusst ist, dass es sich um eine asymmetrische Tauschbeziehung handelt (vgl. Voswinkel 2005).

Vorgefunden haben wir dieses Interaktionsmuster in hochspezialisierten Industrieunternehmen mit kaum mehr als 100 Beschäftigten, die sich erfolgreich in industrielle Marktnischen eingenistet haben zum Teil sogar (trotz ihrer geringen Größe) im globalen Maßstab. Diese Unternehmen erzielen Wettbewerbsvorteile durch hohe Qualitätsstandards sowie flexible und termingerechte Auftragsabwicklung. Der Kostenfaktor spielt dagegen kaum eine Rolle. Sie sind deshalb auf eine hochqualifizierte Belegschaft zumeist männlicher Facharbeiter angewiesen, die bereit sind, sich den Flexibilitätsanforderungen des Unternehmens anzupassen.
Prägend für diese Unternehmen ist die Spannung von Nähe und Distanz zum industriellen Kernbereich sowohl in geografischer als auch in kultureller Hinsicht. Die von uns untersuchten Unternehmen dieses Typus gehören zu zwei traditionellen ländlichen Industriedistrikten, einerseits zum fränkischen Raum um Nürnberg, andererseits zum Bergischen Land mit seiner Nähe zur Rhein-Ruhr-Region. Die Unternehmen sind zwar nicht tarifgebunden, aber die Nähe zu einer Welt, in der Tarifverträge und Betriebsräte selbstverständlich sind, ist für die innerbetriebliche Interaktion entscheidend. Es handelt sich um „Trittbrettfahrer des Tarifsystems" (Artus 2005, S. 394f.). Auch wenn sie formal an keinen Tarifvertrag gebunden sind, orientieren sie sich irgendwie „am Tarif“.

Die Orientierung „am Tarif" kann zwar bedeuten - und das ist vielfach der Fall -, dass Tarifnormen in Bezug auf Grundgehälter und Arbeitszeiten unterschritten werden. Aber der Fortbestand von Besitzständen wie Weihnachts- und Urlaubsgeld ist ein Grundelement für die Legitimität der innerbetrieblichen Austauschbeziehungen. Die Unternehmensleitungen verweisen zudem darauf, dass Abweichungen aufgrund von Öffnungsklauseln auch in tarifgebundenen Unternehmen möglich sind.

Ansonsten gilt das do-ut-des-Prinzip: Zugeständnisse in einem Bereich müssen durch Vergünstigungen in anderen Bereichen ausgeglichen werden. Dies lässt sich sehr gut am Beispiel Arbeitszeit veranschaulichen. Zeitliche Flexibilität stellt einen entscheidenden Faktor für die Sicherung der Wettbewerbsfähigkeit des Unternehmens dar. Kurzfristig anberaumte Überstunden sind nicht ungewöhnlich und werden von den Beschäftigten bereitwillig als Bestandteil guter Arbeit und als Loyalitätsbeweis erbracht. Diese Bereitschaft beruht aber auch darauf, dass es den Beschäftigten umgekehrt möglich ist, kurzfristig Urlaub zu nehmen. Wichtig ist - wie bei anderen Trade-offs - der informelle Charakter. Entscheidend ist, dass das Ansetzen von Überstunden und das Urlaub-Nehmen kurzfristig und unkompliziert erfolgen können.

Der informelle, auf Vertrauen basierende Charakter der Austauschbeziehungen ist auch ein Grund für das Fehlen eines Betriebsrates. Auf Seiten der Unternehmensleitung ist das wichtigste Argument gegen einen Betriebsrat, dass er die Ent- 
scheidungsprozesse verzögere und damit der zeitlichen Flexibilität des Unternehmens schade. Darüber hinaus wissen sie genau, dass ein Betriebsrat die Machtposition der Belegschaft verbessern und den Entscheidungsspielraum des Managements einschränken würde. Da sie zugleich die Position der Belegschaft als Gegenüber in der innerbetrieblichen Tauschbeziehung anerkennen, versuchen sie nicht, einen Betriebsrat mit allen (auch unerlaubten) Mitteln zu verhindern, sondern bieten den Beschäftigten stattdessen andere, informelle Formen der Interessenvertretung an. In zwei der Fallstudien-Betriebe gab es deshalb gewählte Belegschaftssprecher, in einem weiteren Betrieb haben die Teamsprecher der einzelnen Arbeitsgruppen eine ähnliche Funktion übernommen. In den beiden ersten Fällen fehlt diesen Gremien jegliche formale Fixierung. Es gibt weder eine schriftliche Wahlordnung noch eine Wahlperiode. Einmal gewählt, behalten die Belegschaftssprecher ihre Funktion, solange sie von beiden Seiten als solche anerkannt werden.

Auch auf Seiten der Beschäftigten ist der informelle Charakter der Tauschbeziehungen ein entscheidender Grund, warum ein Betriebsrat zwar immer wieder ein Thema ist, es letztendlich aber doch nicht zur Gründung eines Betriebsrates kommt. Sie wissen genau, dass die Geschäftsführung gegen einen Betriebsrat ist. Bevor sie durch eine Betriebsratsgründung einen größeren Konflikt provozieren, wägen sie die Vorteile, die sie durch informelle Arrangements (etwa in Bezug auf die Arbeitszeit oder auf die Nutzung von Maschinen für private Zwecke) haben, gegen die Chancen und Risiken einer Betriebsratsgründung ab. Letztendlich bleibt auch die nichtgenutzte Option einer Betriebsratsgründung Teil der innerbetrieblichen Tauschbeziehungen. Der Bezug auf eine Welt, in der Tarifverträge gelten und Betriebsräte existieren, ist für die Legitimation dieses Systems informellen Tauschs konstitutiv, auch wenn ein Vergleich der Vor- und Nachteile hypothetisch bleiben muss. Umso brüchiger der tarifgebundene und mitbestimmte industrielle Kernbereich wird, desto schwieriger wird auch die Situation für die Beschäftigten im Interaktionsmodus von Anerkennung und Loyalität.

\section{Patronage und Pragmatis- mus in mittelständischen Unternehmen}

Im Unterschied zu den anderen Interaktionsmustern lässt sich dieses Muster nicht einer bestimmten Branchen- oder Qualifikationsstruktur zuordnen. Es findet sich sowohl in Industrie- als auch in Dienstleistungsunternehmen, sowohl bei gering qualifizierten als auch bei hoch qualifizierten Beschäftigten. Wenn sich eine typische Ausgangskonstellation identifizieren lässt, dann am ehesten anhand der Eigentümerstruktur. Es handelt sich in den meisten Fällen um ehemalige Familienunternehmen, die durch anonyme Investoren wie große Konzerne oder Private-Equity-Gesellschaften übernommen wurden. In diesen Unternehmen trifft eine patriarchal geprägte Firmentradition auf die rationalen, eng kalkulierten Vorgaben des Investors. Wirtschaftlicher Druck wird vor allem durch die Investoren ausgeübt, weniger durch die schwierige Lage auf den Absatzmärkten. Aus dieser Konstellation ergibt sich ein patriarchaler Führungsstil, dem der Spielraum für das paternalistische Element abhanden gekommen ist.

Das Management beansprucht für sich die Rolle einer unbestrittenen Autorität im Unternehmen - ganz im Sinne des französischen Wortes "patron“. Es handelt nach der Devise: „Es kann nur einer Herr im Hause sein". Das Herrschaftsverständnis entspricht dabei dem Typus traditionaler Herrschaft bei Max Weber. „Gehorcht wird nicht Satzungen, sondern [...] Personen“ (Weber 1976, S. 130). Entscheidend sind nicht formalisierte Regeln, sondern die persönliche Führungskompetenz des Vorgesetzten. Formale Regeln können zwar existieren, werden aber flexibel gehandhabt. Entscheidungen werden entweder aufgrund von Faustregeln getroffen, die sich bewährt haben, oder nach eigenem Gutdünken. Auch die Leistungsbewertung der Beschäftigten erfolgt nicht nach objektivierbaren Kriterien, sondern nach der persönlichen Einschätzung, der "Gunst“, die ihnen der Vorgesetzte erweist. Für die Beschäftigten, die diese Gunst genießen, eröffnen sich sogar Aufstiegschancen, weil für die Stellung innerhalb der Hierarchie formale Qualifikationen nur eine geringe Rolle spielen. Die Herrschaftsbeziehung hat deshalb auch etwas von dem im deutschen Begriff der Patronage enthaltenen Aspekt der Günstlingswirtschaft.

Die Beschäftigten gehen pragmatisch mit diesem Führungsstil um. Weil sie wissen, dass offene Konflikte von der Geschäftsführung nicht gerne gesehen werden, suchen sie nach anderen Wegen, ihre Situation zu verbessern. Das beginnt mit der geschickten Verhandlungsführung in den jährlichen Mitarbeitergesprächen oder einer pragmatischen Interpretation der an sie gerichteten Anweisungen. Es kann aber auch zu informellen Absprachen unter den Beschäftigten kommen, etwa um der Geschäftsleitung eine andere Form der Schichteinteilung vorzuschlagen. Alles in allem nehmen sie Einschränkungen bei Arbeitszeiten und Löhnen sowie den autoritären Führungsstil hin, so lange sie der Meinung sind, für sich das Beste aus der Situation herauszuholen.

Ein entscheidendes Element für die Stabilität dieser Form der Sozialbeziehung sind die Aufstiegsmöglichkeiten. Beschäftigte, die sich auf das patronale Spiel einlassen, haben die Möglichkeiten in Positionen aufzusteigen, die ihnen anderswo mit ihrer formalen Qualifikation gar nicht zugänglich wären. Denn ganz im Sinne traditionaler Herrschaft spielen formale fachliche Qualifikationen genauso wenig eine Rolle wie klar abgegrenzte Kompetenzen. Beschäftigte, die sich aus Sicht ihrer Vorgesetzten als zuverlässig erweisen, können auch ohne formale Qualifikation Karriere machen und innerhalb der Hierarchie in verschiedenen, sachlich deutlich unterschiedenen Funktionen eingesetzt werden. Diese Regellosigkeit dient zugleich der Legitimation dieser Herrschaftsform.

Darüber hinaus legitimiert sich Herrschaft in diesem Interaktionsmodus durch das Postulat einer vollständigen Interessenidentität:

„Den Interessengegensatz zwischen Arbeitgebern und Arbeitnehmern gibt es nicht. Ich glaub schon, dass es bei den Mitarbeitern angekommen ist, dass es darum geht, dass der Produktionsstandort erhalten bleibt" (Geschäftsführer eines Werkzeugherstellers).

Gegenüber einer Unternehmensleitung, für die die Vorstellung einer Interessenidentität aller grundlegend ist und die abweichende Interessen schlicht ignoriert, kann es auch keinen legitimen Modus der Interessenvertretung geben. Aus Sicht des Managements sind deshalb informelle For- 
men der Interessenvertretung genauso unerwünscht wie ein Betriebsrat. Jede Form von Kritik wird von vornherein stigmatisiert. Wer dennoch abweichende Interessen artikuliert, wird als Querulant abgestempelt. Für die Beschäftigten bleiben - neben der Exit-Option - zwei Alternativen: Entweder sie weichen Konflikten aus und versuchen pragmatisch Lücken des Systems auszunutzen und sich die Gunst der Vorgesetzten zu erarbeiten. Oder (wenn sie damit keinen Erfolg haben oder ihnen eine solche Haltung von vornherein zuwider ist) es kommt zum offenen Konflikt, der schnell in eine Entlassung mündet. Weil die Artikulation abweichender Interessen an sich illegitim ist, liegt es nahe, gleich zur rechtlich abgesicherten Form der Interessenvertretung zu greifen: dem Betriebsrat.

Allerdings stellt die Gründung eines Betriebsrats aus Sicht der Führungskräfte erst recht einen Tabubruch dar. Weil sie Führung nicht einfach als sachliche Aufgabe wahrnehmen (wie die Führungskräfte im Modus Anerkennung/Loyalität) oder als rationale Strategie mit dem Ziel optimaler Kostenreduktion (wie das gehobene Management im repressiven Modus), sondern als soziale Beziehung, die in ihrer persönlichen Autorität gründet, fassen sie Konflikte - insbesondere die Installation eines Betriebsrates - schnell als persönliche Beleidigung auf. Deshalb ist das Thema Betriebsrat bei diesen Führungskräften in besonderer Weise emotional besetzt.

Die Beschäftigten sind sich sehr bewusst, dass sie mit der Gründung eines Betriebsrats einen Tabubruch begehen. Sie setzen die persönliche Beziehung zum Chef aufs Spiel (vor allem dann, wenn sie zu den Privilegierten zählen, die in seiner Gunst stehen) und müssen mit Entlassung oder zumindest systematischer Benachteiligung rechnen, zumal schon einfache Formen der Interessenartikulation mit Sanktionen belegt werden. Den meisten Beschäftigten ist nicht nur das Risiko zu groß, ihnen erscheinen auch die erwarteten Vorteile zu gering - zumindest zu gering im Vergleich zu dem, was sich durch einen pragmatischen Umgang mit dem System erreichen lässt. Ein Beschäftigter, der als stellvertretender Filialleiter einer KFZ-Werkstattkette die Aufstiegsmöglichkeiten im Rahmen der Patronage genutzt hat, formuliert es so:

„Und für mich ist es so, dass der Betriebsrat, was soll er da großartig durchsetzen, was ich nicht alleine versuchen kann. [...] Heutzutage, wenn eine Firma was durchsetzen will, wie wollen Sie sich dagegen stemmen?" (stellvertretender Filialleiter einer KFZ-Werkstattkette).

Hier zeigt sich, dass die Stabilität der Sozialbeziehungen in Betrieben ohne Betriebsrat auch davon abhängt, als wie effektiv die Beschäftigten die Arbeit von Betriebsräten in anderen Betrieben ansehen.

Dennoch kann es unter bestimmten Bedingungen auch in Unternehmen des Typus Patronage/Pragmatismus zur erfolgreichen Gründung eines Betriebsrats kommen. Unter unseren Fallstudien ist die KFZ-Werkstatt-Kette ein passendes Beispiel, weil es dort Filialen mit und ohne Betriebsrat gibt. Hier haben wir sowohl mit Betriebsräten und Beschäftigten, die versucht haben, einen Betriebsrat zu gründen, als auch mit Beschäftigten einer Filiale ohne Betriebsrat gesprochen. Aufgrund dieser Gespräche lassen sich die Umstände, unter denen es zur Gründung eines Betriebsrates kommt, recht genau beschreiben.

Die erste und wichtigste Voraussetzung ist eine spürbare Verschlechterung der Arbeitsbedingungen z. B. infolge der Übernahme des Unternehmens, die „das Fass zum Überlaufen bringt" und als äußerer Anlass für die Initiative fungiert, einen Betriebsrat zu gründen. Im Falle der KFZWerkstatt-Kette bildet die Übernahme des Unternehmens durch eine Private-EquityGesellschaft diesen äußeren Anlass, der zu einem erhöhten Kostendruck auf das lokale Management und damit zu einer Verschlechterung der Arbeitsbedingungen führte.

\section{Repression und Ohnmacht in der prekären Dienstleistungsarbeit}

Spätestens seitdem die Dienstleistungsgewerkschaft ver.di im Dezember 2004 das "Schwarzbuch Lidl“ (Hamann/Giese 2004) veröffentlichte, sind die prekären Arbeitsbedingungen in deutschen DiscounterKetten und die Schwierigkeiten, unter diesen Umständen einen Betriebsrat zu gründen, in aller Munde. Seitdem vergeht kaum ein Monat, in dem nicht irgendeine Zeitung einen neuen Aspekt dieser prekären Arbeitsverhältnisse aufdeckt.

Aber Lidl und Lebensmittel-Discounter im Allgemeinen sind nur ein Beispiel für den sehr viel größeren Bereich prekärer
Dienstleistungsarbeit, der durch folgende Bedingungen gekennzeichnet ist: riesige Konzerne mit sehr kleinen Filialbetrieben und undurchsichtigen Konzernstrukturen, Arbeitsplätze für überwiegend Teilzeitund geringfügig Beschäftigte ohne besondere formale Qualifikation, die sich aus Personengruppen rekrutieren, die eher aufgrund ihrer Lebensumstände als wegen ihrer Qualifikation auf diese Art von "Jobs“ angewiesen sind: Studierende, Migrantinnen und Migranten, Hausfrauen usw. Vergleichbare Arbeitsbedingungen gibt es im Gastgewerbe (insbesondere Fast-Food), in der Gebäudereinigung, bei Sicherheitsdiensten sowie in personenbezogenen und sonstigen Dienstleistungen.

Auch wissenschaftlich ist dieser Bereich inzwischen breit diskutiert (vgl. etwa Bormann 2007 und Voss-Dahm 2005). An dieser Stelle soll deshalb in aller Kürze auf die besonderen Bedingungen eingegangen werden, die dazu führen, dass ausgerechnet in diesem Bereich, in dem eine effektive Interessenvertretung der Beschäftigten besonders nötig wäre, nur in Ausnahmefällen Betriebsräte existieren.

Schon die äußeren Bedingungen (verstreute und undurchsichtige Unternehmensstrukturen sowie das Überwiegen von Teilzeitbeschäftigung, die keine besondere formale Qualifikation erfordert) machen den Bereich prekärer Dienstleistungsarbeit zu einem schwierigen Terrain für die Gründung von Betriebsräten. Die Unternehmensstruktur ist problematisch, weil in den Betrieben vor Ort eine kleinbetriebliche Sozialstruktur vorherrscht, in der jeder jeden kennt und Konflikte schnell zu persönlichen Auseinandersetzungen führen. Hinzu kommt, dass die Teilzeit-Beschäftigten oft auf ein gutes Verhältnis zum Chef angewiesen sind, um ihre konkreten Arbeitszeiten mit ihren anderen Lebensaufgaben in Einklang zu bringen. So meiden sie Konflikte, um z. B. genau dann frei zu bekommen, wenn es für die Betreuung der Kinder erforderlich ist. Im Unterschied zum echten Kleinbetrieb aber ist der Filialleiter vor Ort auch nur ein ausführendes Organ, das die Vorgaben der Unternehmensleitung in den Arbeitsalltag übersetzen muss. Die Filialleiterinnen und Filialleiter sitzen deshalb zwischen den Stühlen. Oft sind sie es, die eine Betriebsratsgründung initiieren. Die wirklichen Ansprechpartner befinden sich außerhalb des Betriebs. Und je undurchsichtiger die Konzernstruktur ist, desto schwieriger wird es, 
überhaupt den richtigen Ansprechpartner zu finden. Hier zeigt sich, dass der Betrieb für netzwerkförmige Unternehmen kein geeigneter Bezugspunkt ist. Zwar sehen die Tarifverträge für den Einzelhandel die Möglichkeit vor, mehrere Filialen vor Ort für die Betriebsratswahlen zusammenzufassen, aber in den Discounter-Ketten greift diese Regelung nicht.

Darüber hinaus wird eine Solidarisierung durch die besondere Form der Beschäftigung erschwert. Die Teilzeitbeschäftigten haben unterschiedlichen Arbeitszeiten und keine gemeinsamen Pausen. Die Arbeit steht im Rahmen der Lebensorganisation neben vielen anderen Aufgaben, was die Bereitschaft, „Kompromisse“ einzugehen, noch verstärkt. Weil keine besonderen formalen Qualifikationen erforderlich sind, sind die Beschäftigten leicht ersetzbar. Es entsteht ein Gefühl der Ohnmacht, das dazu führt, dass in Konfliktfällen die Exit-Option oft näher liegt als die Voice-Option.

Ist es für die Beschäftigten aufgrund dieser äußeren Bedingungen schon schwierig genug, ihre Interessen zu vertreten, werden Ansätze dazu zusätzlich durch eine repressive Unternehmenspolitik im Keim erstickt. Zwar lassen sich auch in diesem Interaktionsmuster zwei Führungsstrategien unterscheiden, aber für die Möglichkeiten einer effektiven Interessenvertretung machen sie kaum einen Unterschied. Insbesondere amerikanische Unternehmen, die in diesem Bereich tätig sind, setzen auf eine starke Corporate Identity und eine symbolische Politik der Anerkennung. Diese Variante des repressiven Interaktionsmusters bezeichnet Ingrid Artus (2008) als repressive Integration. Zwar führt eine Politik der Anerkennung zu einer größeren $\mathrm{Zu}$ friedenheit und zu einer stärkeren Identifikation mit dem Unternehmen, aber die objektiven Bedingungen für die Gründung eines Betriebsrats sind genauso schlecht wie in den anderen Unternehmen dieses Bereichs und der Widerstand der Unternehmensleitungen ist genauso hart. Außerdem wird die Artikulation abweichender Interessen schnell als Ausscheren aus der Unternehmenscommunity interpretiert. Andere Unternehmen, insbesondere die großen deutschen Discounter-Ketten, verzichten dagegen selbst auf symbolische Formen der Anerkennung: kein freundliches Wort, kein Lob, keine Auszeichnungen. Stattdessen werden Beschäftigte von ihren Vorgesetzten angeschrieen, beleidigt oder mit Abmahnungen bedroht. Es herrscht eine Kultur des Misstrauens.

Spätestens, wenn es zur Gründung eines Betriebsrates kommt, unterscheiden sich beide Varianten nicht mehr. Die Unternehmensleitungen versuchen mit allen legalen und illegalen Mitteln, eine Betriebsratsgründung zu verhindern: Die Initiatoren werden entlassen, auch wenn die Entlassung vor dem Arbeitsgericht anschließend für nichtig erklärt wird. Oder es wird ihnen Geld geboten, wenn sie auf die Betriebsratswahl verzichten. Die übrigen Beschäftigten werden unter Druck gesetzt. Wenn sich eine Betriebsratswahl nicht mehr verhindern lässt, versucht die Unternehmensleitung, die Betriebsräte mit loyalen Personen zu besetzen.

Trotzdem gibt es auch erfolgreiche Betriebsratsgründungen im Bereich prekärer Dienstleistungsarbeit - insbesondere dort, wo die einzelnen Betriebe nicht ganz so klein sind. Für zwei unserer Fallstudien trifft dies zu. In dem Paketdienst haben die einzelnen Betriebe mehrere hundert Beschäftigte. Auch die Einzelhandelskette, in der wir eine Intensivfallstudie durchgeführt haben, verfügt neben kleinen Discountermärkten über größere Supermärkte mit 100 bis 200 Beschäftigten, von denen einige zudem von Konkurrenten übernommen wurden und aus dieser Zeit noch einen Betriebsrat haben. In diesen Unternehmen gibt es Betriebe mit und ohne Betriebsrat. Die Unternehmensleitungen reagieren auf diese Situation damit, dass sie die Bereiche, in denen Betriebsräte existieren, voneinander isolieren. Wenn es schon Betriebsräte gibt, sollen wenigstens keine Gesamtbetriebsräte entstehen - geschweige denn eine flächendeckende Struktur von Betriebsräten.

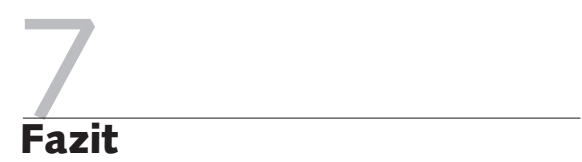

Aus dem Vergleich dieser Konstellationen der Betriebsratlosigkeit und der darin existierenden Interaktionsmodi lassen sich einige Schlussfolgerungen ableiten:

(1) Das Bild von einem tarifgebundenen und mitbestimmten Kern und einer Peripherie, in der Tarifverträge und Betriebsräte nur teilweise oder gar nicht existieren, hat nur noch für das Interaktionsmuster
Anerkennung/Loyalität Bedeutung. Insgesamt gilt eher das Bild einer Segmentation der industriellen Beziehungen. Es gibt genügend Bereiche, die einer eigenen Gesetzmäßigkeit folgen und für die die in der Tarifautonomie und dem Betriebsverfassungsgesetz verankerten Institutionen nicht einmal eine entfernte Norm oder Normalität darstellen.

(2) „Alternative Gremien“ spielen in den von uns untersuchten Unternehmen nur eine geringe Rolle (vgl. Hauser-Ditz et al. in diesem Heft). Das liegt einerseits daran, dass die Konflikte systematisch individualisiert werden (Einbindung/Autonomie und Patronage/Pragmatismus), andererseits daran, dass die Fronten zu sehr verhärtet sind und Mitsprache von Seiten der Unternehmensleitung grundsätzlich unerwünscht ist (Patronage/Pragmatismus und Repression/Ohnmacht). Im Interaktionsmodus Einbindung/Autonomie gibt es zwar vom Management initiierte Gremien, aber sie haben keinen kontinuierlichen Charakter, sondern werden ad hoc für einen konkreten Anlass gebildet. Ansonsten finden sich informelle Organe der Interessenvertretung nur in den Unternehmen, die sich dem Interaktionsmuster Anerkennung/Loyalität zuordnen lassen. Es ist das einzige Muster, in dem auch die Führungskräfte von einem kollektiven Interessengegensatz zwischen Beschäftigten und Unternehmensleitung ausgehen, der irgendwie geregelt werden muss.

(3) Dass es im Bereich prekärer Dienstleistungsarbeit so schwierig ist, Betriebsratsstrukturen zu etablieren, obwohl sie hier besonders nötig wären, liegt nicht allein an der Ohnmacht der Beschäftigten, sondern auch daran, dass der Betrieb aufgrund der netzwerkartigen Unternehmensstruktur keine sinnvolle Bezugsgröße ist.

(4) Trotz aller gesetzlichen Bestimmungen praktizieren die Unternehmensleitungen in den beiden Mustern Patronage/Pragmatismus und Repression/Ohnmacht systematische Strategien der Betriebsratsverhinderung. Hier erweisen sich die gesetzlichen Regelungen als zahnlos. Lieber nehmen die Unternehmensleitungen Prozesse vor dem Arbeitsgericht in Kauf als einen Betriebsrat zuzulassen. 
Artus, I. (2005): Betriebe ohne Betriebsrat: Gelände jenseits des Tarifsystems, in: WSI-Mitteilungen 7, S. 392-397

Artus, I. (2008): Prekäre Vergemeinschaftung und verrückte Kämpfe. Repressive Integration als Herrschaftsmodus im prekären Dienstleistungsbereich, in: Prokla 150, S. 27-48

Boes, A./Baukrowitz, A. (2002): Arbeitsbeziehungen in der IT-Industrie. Erosion oder Innovation der Mitbestimmung?, Forschung aus der HansBöckler-Stiftung 37, Berlin

Böhm, S./Lücking, S. (2006): Orientierungsmuster des Managements in betriebsratslosen Betrieben - Zwischen Willkürherrschaft und Human Resource Management, in: Artus, I./Böhm, S./Lücking, S./Trinczek, R. (Hrsg.): Betriebe ohne Betriebsrat. Informelle Interessenvertretung in Unternehmen, Frankfurt a. M./New York, S. 107-139

Bormann, S. (2007): Angriff auf die Mitbestimmung. Unternehmensstrategien gegen Betriebsräte - der Fall Schlecker, Berlin
Ellguth, P./Kohaut, S. (2007): Tarifbindung und betriebliche Interessenvertretung. Aktuelle Ergebnisse aus dem IAB-Betriebspanel 2006, in: WSIMitteilungen 9, S. 511-514

Hamann, A./Giese, G. (2004): Schwarz-Buch Lidl: billig auf Kosten der Beschäftigten, Berlin

Voss-Dahm, D. (2005): Verdrängen Minijobs „normale“ Beschäftigung? Warum die Neuregelung der geringfügigen Beschäftigung erfolgreich und zugleich problematisch ist: das Beispiel des Einzelhandels, in: Institut Arbeit und Technik: Jahrbuch 2005, S. 232-246

Voswinkel, S. (2005): Reziprozität und Anerkennung in Arbeitsbeziehungen, in: Adloff, F./Mau, S. (Hrsg.): Vom Geben und Nehmen. Zur Soziologie der Reziprozität, Frankfurt/M., S. 237-256

Weber, M. (1976): Wirtschaft und Gesellschaft, Studienausgabe, Tübingen 